\title{
Pedestal structure and inter-ELM evolution for different main ion species in ASDEX
}

\section{Upgrade}

F. M. Laggner, ${ }^{1, a)}$ E. Wolfrum, ${ }^{2}$ M. Cavedon, ${ }^{2,3}$ F. Mink, ${ }^{2,3}$ M. Bernert, ${ }^{2}$ M. G. Dunne, ${ }^{2}$ P. A. Schneider, ${ }^{2}$ A. Kappatou, ${ }^{2}$ G. Birkenmeier, ${ }^{2,3}$ R. Fischer, ${ }^{2}$ M. Willensdorfer, ${ }^{2}$ F. Aumayr,${ }^{1}$ the EUROfusion MST1 Team, ${ }^{\text {b) }}$ and the ASDEX Upgrade Team

1) Institute of Applied Physics, TU Wien, Fusion@ÖAW, Wiedner Hauptstr. 8-10, 1040 Vienna, Austria

2) Max Planck Institute for Plasma Physics, Boltzmannstr. 2, 85748 Garching, Germany

3) Physik-Department E28, Technische Universität München, James-Franck-Str. 1, 85748 Garching, Germany

(Dated: 22 December 2016) 
Pedestal structure and inter-ELM evolution for different main ion species

In tokamak plasmas with different main ion species a change of confinement occurs, known as isotope effect. Experiments comparing hydrogen (H), deuterium (D) and helium $\left({ }^{4} \mathrm{He}\right)$ plasmas have been performed to identify processes that define the pedestal structure and evolution in between the crashes of edge localized modes (ELMs). The pedestal top electron densities and temperatures have been matched to compare the pedestal shape and stability. In the $\mathrm{D}$ and $\mathrm{H}$ discharges the pedestal electron temperature profiles do not differ, whereas the density profile in $\mathrm{H}$ has shallower gradients. Furthermore, the heat flux across the pedestal in $\mathrm{H}$ is roughly a factor of two higher than in D. In ${ }^{4} \mathrm{He}$ plasmas at similar stored energy the pedestal top electron density is roughly a factor of 1.5 larger than in the references owing to the larger effective charge. Peeling-ballooning theory, which is independent of the main ion species mass, can sufficiently describe the pedestal stability in the hydrogenic plasmas. The inter-ELM pedestal evolution has the same sequence of recovery phases for all investigated species, giving evidence that similar mechanisms are acting in the pedestals. This is further supported by a similar evolution of the inter-ELM magnetic signature as well as corresponding toroidal structure.

PACS numbers: 52.55.-s, 52.55.Fa, 52.55.Tn

Keywords: Plasma, Tokamak, ELM

a) Electronic mail: laggner@iap.tuwien.ac.at

b) See the author list of "Overview of progress in European Medium Sized Tokamaks towards an integrated plasma-edge/wall solution" by H. Meyer et al., to be published in Nuclear Fusion Special issue: Overview and Summary Reports from the 26th Fusion Energy Conference (Kyoto, Japan, 17-22 October 2016) 
Pedestal structure and inter-ELM evolution for different main ion species

\section{INTRODUCTION}

The next step fusion experiment, ITER, will start its operation with either hydrogen (H) or more likely helium $\left({ }^{4} \mathrm{He}\right)$ plasmas to commission the operationally relevant systems and plasma diagnostics in a non-nuclear environment and develop plasma scenarios for deuterium-tritium (D-T) operation ${ }^{1}$. The foreseen plasma scenario is the high confinement mode (H-mode), which is characterized by an edge transport barrier (ETB) and connected to steep gradients in the temperature, density and consequently in the pressure at the plasma edge, called pedestal. The origin of the ETB is a radially sheared $\mathrm{E} \times \mathrm{B}$ flow, caused by the radial electric field $\left(E_{\mathrm{r}}\right)$, which reduces the turbulent transport due to decorrelation of the turbulent eddies ${ }^{213}$. To achieve the transition from low confinement mode (L-mode) to H-mode the L-H power threshold $\left(P_{\mathrm{L}-\mathrm{H}}\right)$, has to be exceeded ${ }^{4}$. This $P_{\mathrm{L}-\mathrm{H}}$ differs when exchanging the main ion species of the plasma ${ }^{5}$. It has been found that the edge ion heat flux at the L-H transition is about twice as high in $\mathrm{H}$ as in deuterium (D) plasmas ${ }^{6}$ at similar ion temperature gradient $\left(\nabla T_{\mathrm{i}}\right)$. The latter suggests that in both plasmas $E_{\mathrm{r}}$ at the L-H transition is similar.

Similar to the difference in $P_{\mathrm{L}-\mathrm{H}}$, better plasma confinement for heavier hydrogenic isotopes has been experimentally observed in $\mathrm{H}$-mode ${ }^{78}$. This behaviour is contrary to the expected confinement assuming gyro-Bohm diffusive transport because the gyro radius increases with mass at fixed temperature. The worse energy confinement in H H-mode plasmas was attributed to enlarged core heat conductivity ${ }^{9}$. Also losses due to edge localized modes (ELMs) and the pedestal can have a significant contribution to the plasma stored energy $\left(W_{\mathrm{MHD}}\right)^{10}$. The behaviour of ELMs, in terms of ELM frequency $\left(f_{\mathrm{ELM}}\right)$, particle and energy losses, has been reported to change, when different main ion species are investigated ${ }^{11}$ especially in ${ }^{4} \mathrm{He}$ plasmas 1213 . With respect to ITER's non-nuclear operation one important question is if the behaviour of $\mathrm{H}$ or ${ }^{4} \mathrm{He}$ plasmas can be extrapolated to scenarios for D-T operation.

Usually core, edge and effects caused by ELMs are coupled ${ }^{1415}$, this paper focuses on their separation and ordering in plasmas with different main ion species. For this reason, the pedestal top electron density $\left(n_{\mathrm{e}}\right)$ and electron temperature $\left(T_{\mathrm{e}}\right)$ in $\mathrm{D}, \mathrm{H}$ and ${ }^{4} \mathrm{He}$ plasmas were matched. Using the plasma edge diagnostics with high spatial as well as temporal resolution at ASDEX Upgrade ${ }^{\sqrt{16}}$, the pedestal structure, stability and inter-ELM evolution 
Pedestal structure and inter-ELM evolution for different main ion species

are characterized.

The paper is organized as follows: The experimental procedure is motivated and an introduction to the utilized data analysis methods is presented in Section II. The pedestals of hydrogenic species plasmas $(\mathrm{D}, \mathrm{H})$ are compared to linear ideal magnetohydrodynamic (MHD) stability analysis and the inter-ELM evolution is characterized in Section III. Section IV] shows pedestal structure and inter-ELM evolution of ${ }^{4} \mathrm{He}$ plasmas. Finally, the results are summarized in Section V] giving a consistent picture: peeling-ballooning (PB) theory consistently describes the pedestal stability for the investigated cases of hydrogenic species. ELM behaviour and pedestal structure for different main ion species can be explained by the necessary variation of control parameters. There seems to be no difference in the pedestal dynamics, magnetic signature and corresponding structure of inter-ELM magnetic fluctuations, indicating no change of the dominant processes by exchange of the main plasma isotope.

\section{EXPERIMENTAL MOTIVATION AND SETUP}

The conducted experiments aim at a quantitative comparison of pedestals in plasmas with different main ion species. A match of the pedestal $n_{\mathrm{e}}, T_{\mathrm{e}}$ and ion temperature $\left(T_{\mathrm{i}}\right)$ was performed keeping the magnetic configuration; toroidal magnetic field $\left(B_{\mathrm{t}}\right)$, plasma current $\left(I_{\mathrm{p}}\right)$ and plasma shape. The chosen plasma scenario was a lower single null discharge with $I_{\mathrm{p}}=1.0 \mathrm{MA}, B_{\mathrm{t}}=-2.5 \mathrm{~T}$ (negative sign stands for opposite direction to $I_{\mathrm{p}}$ ) at an average triangularity of 0.22 . The varied control parameters were heating power $\left(P_{\text {heat }}\right)$ and the gas puff. This procedure enables a direct comparison of the edge stability between the different ion species. The ELM behaviour (frequency, losses and temporal evolution) with similar pre-ELM pedestal structure can be directly compared and the inter-ELM magnetic activity is characterized.

The pedestal profiles are measured by the following diagnostics: The $n_{\mathrm{e}}$ profile is measured by lithium beam emission spectroscopy ${ }^{17}$ at the edge and by deuterium cyanide laser interferometry ${ }^{18}$ in the core. The $T_{\mathrm{e}}$ profile is reconstructed from electron cyclotron emission $(\mathrm{ECE})^{19}$. Within the integrated data analysis framework ${ }^{20}$ the information of these diagnostics is combined to evaluate the best fits of $n_{\mathrm{e}}$ and $T_{\mathrm{e}}$ (parameterized by cubic splines). Here, a forward modelling of the electron cyclotron radiation transport ${ }^{21}$ is ap- 
Pedestal structure and inter-ELM evolution for different main ion species

plied to reliably determine the $T_{\mathrm{e}}$ profile at the pedestal and around the separatrix in the optically thin plasma. The $T_{\mathrm{e}}$ profile is radially shifted such that a separatrix $T_{\mathrm{e}}$ of $100 \mathrm{eV}$ is matched ${ }^{16}$. Relative alignment of the $n_{\mathrm{e}}$ and $T_{\mathrm{e}}$ profiles is cross checked with the Thomson scattering diagnostic ${ }^{22}$, which measures both quantities simultaneously at the same position. To achieve these conditions, generally shifts smaller than $3 \mathrm{~mm}$ are applied. The later presented $n_{\mathrm{e}}$ and $T_{\mathrm{e}}$ profiles are analyzed with a temporal resolution of $250 \mu \mathrm{s}$.

The presented $T_{\mathrm{i}}$ profiles are measured by the edge charge exchange recombination spectroscopy (CXRS) system ${ }^{23}$ with a temporal resolution of $2.3 \mathrm{~ms}$. The radial resolution is $3 \mathrm{~mm}$ at the edge and increases towards the pedestal top to roughly $5 \mathrm{~mm}$. Typically, the CXRS diagnostic employs charge exchange between low charge number impurities, e.g. boron or nitrogen, and injected neutrals. In the case of ${ }^{4} \mathrm{He}$ plasmas the main ions can be directly investigated. A recent upgrade of the spectrometer ${ }^{24}$ enables a temporal resolution down to $70 \mu$ s enabling the study of the inter-ELM evolution of $T_{\mathrm{i}}$.

\section{COMPARISON OF HYDROGENIC MAIN ION SPECIES}

\section{A. Pedestal structure and stability}

By a variation of two control parameters, gas puff and $P_{\text {heat }}$, a match of $T_{\mathrm{e}}$ as well as reasonable agreement of $T_{\mathrm{i}}$ pedestal profiles (pedestal top $T_{\mathrm{i}}$ slightly higher in $\mathrm{H}$ ) are achieved (see Fig. 1, blue: D, red: H). Due to a different electron density gradient $\left(\nabla n_{\mathrm{e}}\right)$ it is only possible to match the pedestal top $n_{\mathrm{e}}$ Fig. 1 a . This pedestal top match required roughly a factor of 2 higher heating power (D: $P_{\text {heat }} 3.9 \mathrm{MW}, \mathrm{H}: P_{\text {heat }} 7.5 \mathrm{MW}$ ) as well as a factor of almost 10 higher gas puff (D: $\left.1.5 \cdot 10^{21} \mathrm{e} / \mathrm{s}, \mathrm{H}: 12.1 \cdot 10^{21} \mathrm{e} / \mathrm{s}\right)$ in the $\mathrm{H}$ plasma. The heating systems were electron cyclotron resonance heating (ECRH) at central deposition location to avoid impurity accumulation (1.1 MW in both plasmas) and neutral beam injection (NBI) operated with the particular ion species. The shallower $\nabla n_{\mathrm{e}}$ in the $\mathrm{H}$ plasma is probably caused by the change of particle confinement. A stronger ionization source in $\mathrm{H}$ due to the higher gas puff, located further inside the plasma because of the faster velocity of neutrals, would lead to higher pedestal top $n_{\mathrm{e}}$ at similar particle confinement.

To compare the stability of both plasmas and investigate the impact of the shallower $\nabla n_{\mathrm{e}}$, linear MHD stability analysis is performed using the recently improved stability workflow 

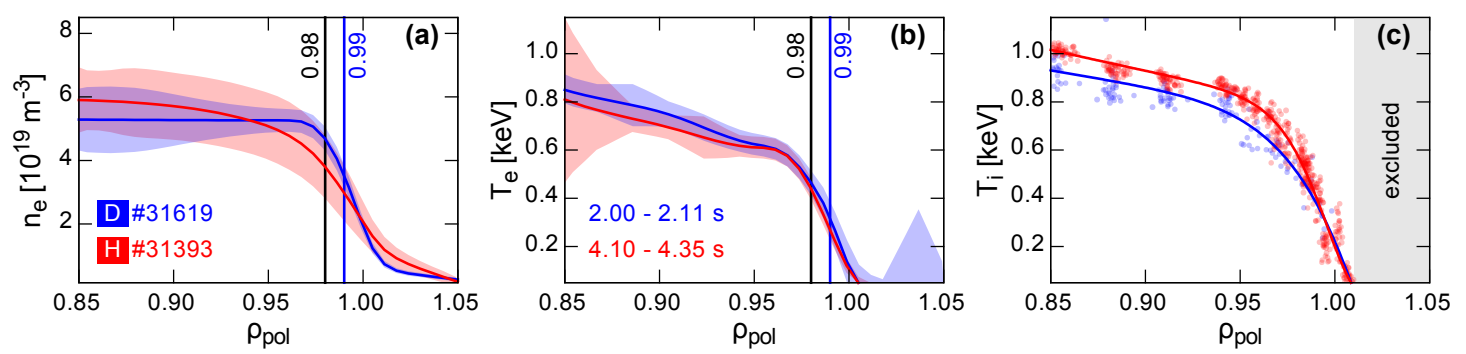

FIG. 1: Pre-ELM (averaged between -2.0 and $-1.0 \mathrm{~ms}$ relative to the ELM onset) profiles of (a) $n_{\mathrm{e}}$, (b) $T_{\mathrm{e}}$ and (c) $T_{\mathrm{i}}$ for $\mathrm{D}$ (blue) and $\mathrm{H}$ (red). The coloured vertical bars indicate radial positions at which the inter-ELM temporal evolution of the corresponding quantities are tracked in Fig. 4 . The gradient of the $n_{\mathrm{e}}$ profile in $\mathrm{H}$ is shallower than in $\mathrm{D}$, leading to a wider pedestal and a reduction of the achievable pressure gradient (compare Fig. 2).

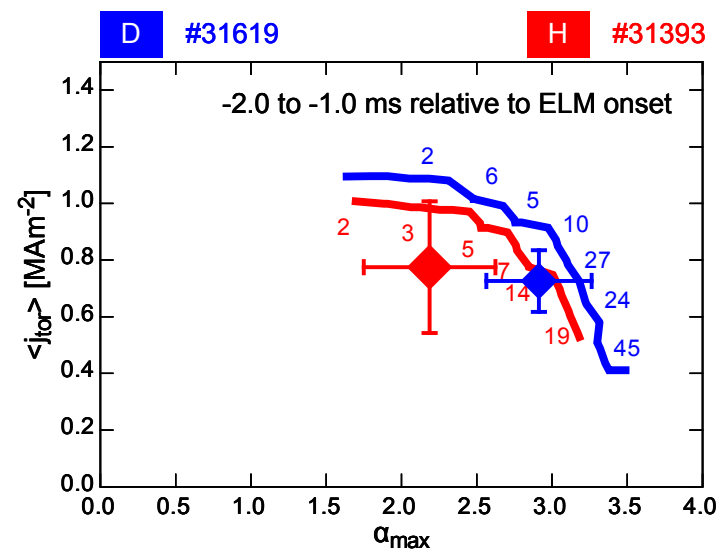

FIG. 2: Comparison of the pre-ELM stability boundaries (determined between -2 and $-1 \mathrm{~ms}$ relative to the ELM onset) for the D (blue) and the $\mathrm{H}$ (red) plasma: average toroidal current density in the pedestal $\left(\left\langle j_{\text {tor }}\right\rangle\right)$ and maximum normalized pressure gradient $\left(\alpha_{\max }\right)$. Both operational points are located close to the PB part of the boundary.

at ASDEX Upgrade ${ }^{25}$. As input served a pressure constrained magnetic equilibrium that includes the reconstructed edge current density 26 . The resulting boundaries and corresponding operational points are presented in Fig. 2 in terms of average toroidal current density in the pedestal $\left(\left\langle j_{\text {tor }}\right\rangle\right)$ and maximum normalized pressure gradient $\left(\alpha_{\max }\right)$. Owing to the shallower $\nabla n_{\mathrm{e}}$ in $\mathrm{H}, \alpha_{\max }$ is lower, leading to a reduced operational point in $\alpha_{\max }$. Within the experimental uncertainties both plasmas are qualitatively consistent with PB theory. This suggests that the pedestal stability is not affected by the main ion species and simply 

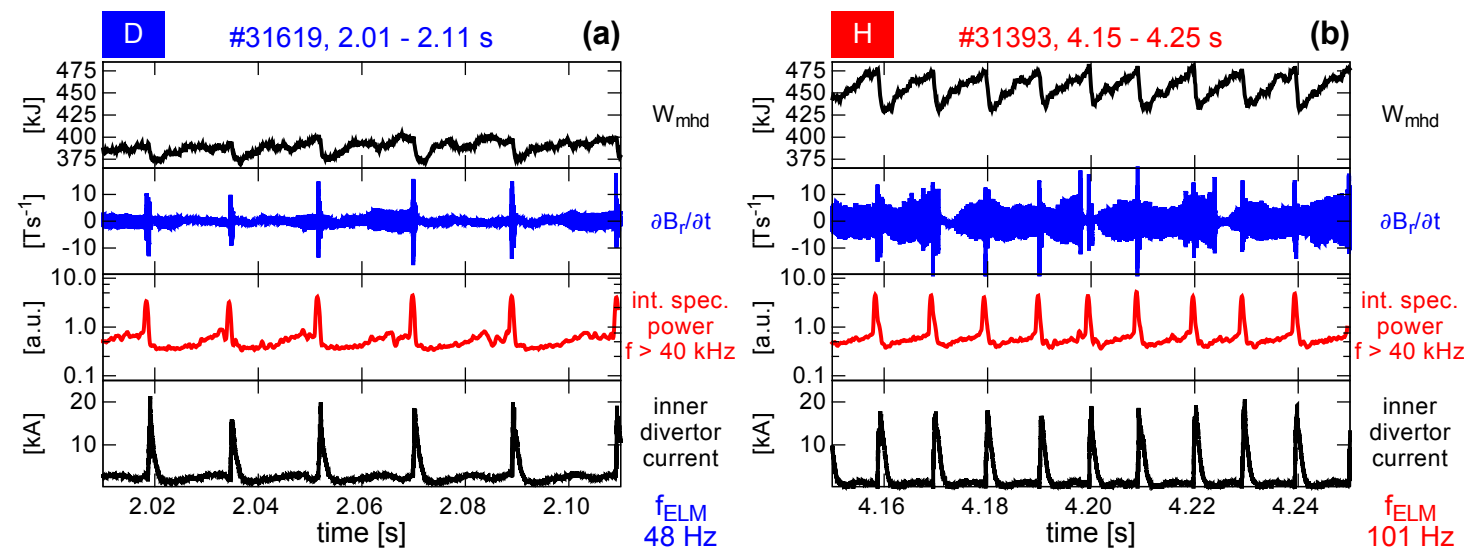

FIG. 3: Time traces of $100 \mathrm{~ms}$ duration of the a $\mathrm{D}$ and $\mathrm{b}$ H plasma discharge: plasma stored energy ( $W_{\mathrm{MHD}}$, black, top plot), radial magnetic field fluctuations $\left(\partial B_{\mathrm{r}} / \partial t\right.$, blue),

the integrated spectral power of $\partial B_{\mathrm{r}} / \partial t$ for frequencies larger than $40 \mathrm{kHz}$ (red) and divertor shunt current (black, bottom plot). The $f_{\mathrm{ELM}}$ in the $\mathrm{H}$ plasma is about twice as large as in the D plasma and the $W_{\mathrm{MHD}}$ losses due to ELMs are larger in $\mathrm{H}$.

determined by the plasma edge profiles.

\section{B. ELM behaviour and losses}

The evolution in between ELM crashes is compared using the time traces presented in Fig. 3 for $\mathrm{D}$ and $\mathrm{H}$. The plasma stored energy $\left(W_{\mathrm{MHD}}\right)$, radial magnetic field fluctuations $\left(\partial B_{\mathrm{r}} / \partial t\right)$ measured by a magnetic pickup coil that is located at the low field side (LFS) midplane (B31-14), the integrated spectral power of $\partial B_{\mathrm{r}} / \partial t$ (for frequencies larger than $40 \mathrm{kHz}$ ) and divertor current, measured by shunts in the divertor target plates (bursts indicate the ELM crashes), are plotted. By counting the number of ELMs (since the chosen time intervals have similar length), it is visible that the $f_{\mathrm{ELM}}$ is about twice as large in the $\mathrm{H}$ plasma in comparison to the D plasma. A list of further ELM characterizing quantities and their uncertainties can be found in Table I. In terms of average ELM energy losses $\left(\Delta W_{\mathrm{MHD}}\right)$ the ELMs in $\mathrm{H}$ are almost twice as large as in D. But the $W_{\text {MHD }}$ before the ELM crash is about $20 \%$ higher in $\mathrm{H}$ because of slightly higher $n_{\mathrm{e}}$ and $T_{\mathrm{i}}$ from the pedestal inwards (compare Fig. 1) and likely a larger amount of fast-ions since the heating power from NBI is higher. In principle the larger stored energy should be beneficial for the pedestal stability due to a bigger Shafranov shift. However, it can be seen in Fig. 2 this effect is overruled by 
Pedestal structure and inter-ELM evolution for different main ion species

\begin{tabular}{|c|c|c|c|c|c|c|c|c|c|}
\hline species & shot & $f_{\mathrm{ELM}}[\mathrm{Hz}]$ & $\Delta W_{\mathrm{MHD}}[\mathrm{kJ}]$ & $\Delta W_{\mathrm{MHD}}[\%]$ & $P_{\text {ELM }}[\mathrm{MW}]$ & $P_{\text {heat }}[\mathrm{MW}]$ & $P_{\text {net }}[\mathrm{MW}]$ & $P_{\text {rad,sep }}[\mathrm{MW}]$ & $\frac{P_{\mathrm{ELM}}}{P_{\mathrm{net}}-P_{\mathrm{rad}, \mathrm{sep}}}[\%]$ \\
\hline $\mathrm{D}$ & \#31619 & $48 \pm 17$ & $20.1 \pm 4.6$ & $5.1 \pm 1.2$ & $0.97 \pm 0.41$ & 3.93 & 3.76 & $1.25 \pm 0.15$ & $38.6 \pm 16.5$ \\
\hline $\mathrm{H}$ & \#31393 & $101 \pm 6$ & $36.9 \pm 5.2$ & $7.9 \pm 1.1$ & $3.73 \pm 0.97$ & 7.51 & 7.28 & $0.72 \pm 0.22$ & $56.8 \pm 8.9$ \\
\hline
\end{tabular}

TABLE I: ELM energy and power losses for D and H: ELM frequency $\left(f_{\mathrm{ELM}}\right)$, average ELM energy loss $\left(\Delta W_{\text {MHD }}\right)$, power loss by ELMs $\left(P_{\text {ELM }}\right)$, heating power $\left(P_{\text {heat }}\right)$, corrected heating power $\left(P_{\text {net }}\right)$, radiated power inside the separatrix $\left(P_{\text {rad,sep }}\right)$ and relative power loss by ELMs $\left[P_{\mathrm{ELM}} /\left(P_{\text {net }}-P_{\text {rad,sep }}\right)\right]$. In both species about half of power crossing the separatrix is transported by ELMs. In absolute numbers an enhanced heat flux across the pedestal in $\mathrm{H}$ is required to balance the power.

the shallower $\nabla n_{\mathrm{e}}$ and only of secondary order in this comparison.

Taking into account the differences in $W_{\mathrm{MHD}}$, the average relative ELM energy losses are approximately 1.5 times higher in $\mathrm{H}$ in comparison to D. Combining $f_{\mathrm{ELM}}$ and $\Delta W_{\mathrm{MHD}}$, the power losses caused by ELMs $\left(P_{\text {ELM }}\right)$ in $\mathrm{H}$ are about 4 times the one in D. The relative power losses caused by ELMs $\left[P_{\text {ELM }} /\left(P_{\text {net }}-P_{\text {rad,sep }}\right)\right]$ are calculated using the for beam losses and $\partial W_{\mathrm{MHD}} / \partial t$ corrected heating power $\left(P_{\text {net }}\right)$ and the radiated power inside the separatrix $\left(P_{\text {rad,sep }}\right)$. These are in the range of about 40 to $50 \%$ in both plasmas. In D, $P_{\text {rad,sep }}$ is 1.8 times larger than in $\mathrm{H}$, because of a higher tungsten concentration since low gas puff and low heating power were applied. To fulfill power balance the remaining power $P_{\text {net }}-P_{\text {rad,sep }}-P_{\text {ELM }}$ has to be lost by heat transport. Therefore, a factor of 1.8 higher heat flux across the pedestal is required in $\mathrm{H}$ in comparison to $\mathrm{D}$.

An indicator for the presence of instabilities or modes in the plasma are fluctuations of the magnetic field. In between the ELM crashes, the detected $\partial B_{\mathrm{r}} / \partial t$ (Fig. 3, blue) is larger in amplitude for $\mathrm{H}$ than for $\mathrm{D}$. This is mainly caused by a larger amplitude response of a core mode in the $\mathrm{H}$ plasma, which dominates the power in frequencies up to $40 \mathrm{kHz}$. The inter-ELM integrated spectral power for frequencies larger than $40 \mathrm{kHz}$ (red) is at similar levels for both main ion species. Furthermore, the integrated spectral power shows a similar evolution. After the ELM crash a phase of low fluctuation level, correlated to the $n_{\mathrm{e}}$ pedestal recovery (see Section III C), is observed. This phase is followed by an increase (during the $T_{\text {e }}$ pedestal recovery), resulting in a saturated phase before the ELM onset, in which it has been found that the maximum pedestal gradients are clamped 27 . The toroidal structure of these fluctuations is analyzed in more detail in Section IIID, 
Pedestal structure and inter-ELM evolution for different main ion species
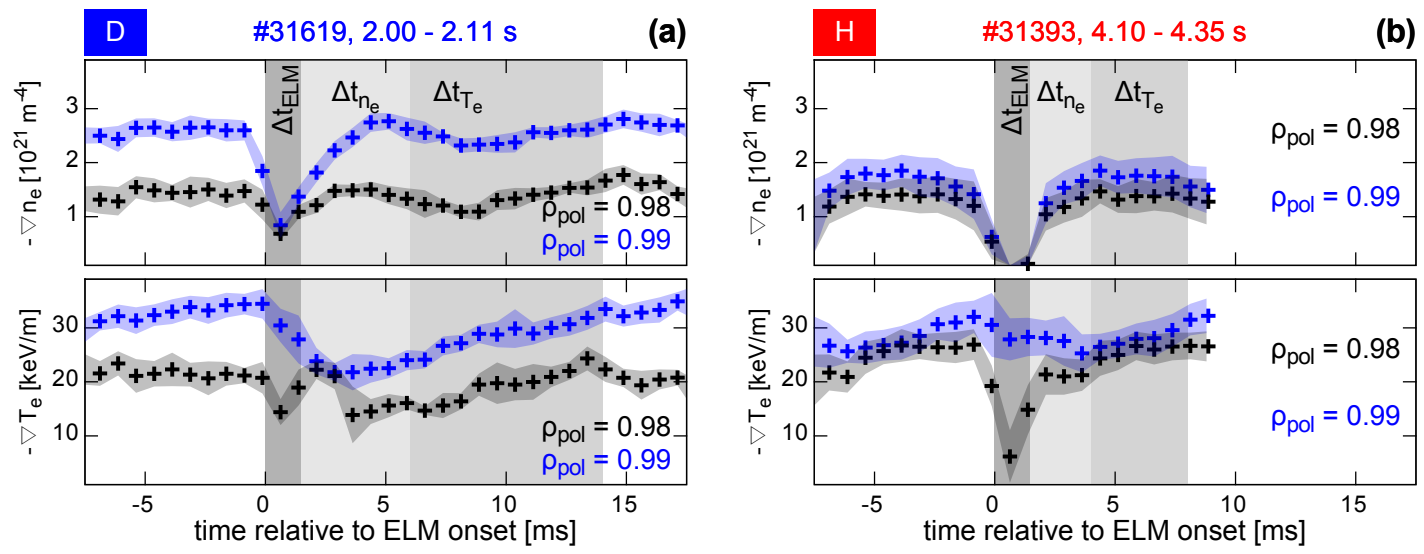

FIG. 4: Pedestal gradient evolution throughout the ELM cycle: $\nabla n_{\mathrm{e}}$ and $\nabla T_{\mathrm{e}}$ at certain positions ( $\rho_{\text {pol }}$; compare Fig. 1) for the (a) D and (b) H plasma. The pedestal recovery phases have a similar sequence in both main ion species but different duration $\left(\Delta t_{n_{\mathrm{e}}}, \Delta t_{T_{\mathrm{e}}}\right)$.

\section{Inter-ELM pedestal evolution}

Comparing the inter-ELM evolution of the pedestal $n_{\mathrm{e}}$ and $T_{\mathrm{e}}$ for the different ion species gives insight in the build-up of the pre-ELM pedestal structure. It has been found previously in $\mathrm{D}$ plasmas that the $n_{\mathrm{e}}$ pedestal recovers on a shorter timescale than the $T_{\mathrm{e}}$ pedestal $\frac{28}{}$. A change of this behaviour in $\mathrm{H}$ would be a strong indication for different mechanisms acting on the pedestal. In Fig. 4 the ELM synchronized evolution of $\nabla n_{\mathrm{e}}$ and electron temperature gradient $\left(\nabla T_{\mathrm{e}}\right)$ for $\mathrm{D}$ and $\mathrm{H}$ at representative radial locations $\left(\rho_{\mathrm{pol}}=0.98\right.$ and 0.99$)$, which are also indicated in Fig. 1, are presented. In both main ion species the pedestal shows qualitatively similar recovery phases, i.e. the pedestal $n_{\mathrm{e}}$ recovers faster than the pedestal $T_{\mathrm{e}}$.

The first phase in the ELM-cycle is the ELM crash, which has a duration $\Delta t_{\text {ELM }}$ of $1.5 \mathrm{~ms}$ for both plasmas, according to the duration of the bursts in the divertor shunt current. This phase is followed by the recovery of the $n_{\mathrm{e}}$ pedestal with duration $\Delta t_{n_{\mathrm{e}}}$, which is longer in D. Two possible mechanisms could cause this difference in $\Delta t_{n_{\mathrm{e}}}$ : On the one hand the 10 times higher gas puff (larger particle source) as well as the faster velocity of recycled particles due to the lower mass in $\mathrm{H}$ or on the other hand an increased outward particle transport from the core to the pedestal top in $\mathrm{H}$. The third phase is the recovery of the $T_{\mathrm{e}}$ pedestal, respectively $\nabla T_{\mathrm{e}}$. Here, the duration $\Delta t_{T_{\mathrm{e}}}$ is also longer in $\mathrm{D}$, most probably caused by the lower heat flux to the pedestal. During the last phase of the ELM cycle the pedestal 
Pedestal structure and inter-ELM evolution for different main ion species

gradients do not evolve and high frequency magnetic fluctuations set in (see Section IIID). This period lasts up to several milliseconds in $\mathrm{D}$, whereas in $\mathrm{H}$ it is just present for 1 to 2 ms. Qualitatively, a similar sequence of pedestal recovery phases is observed in D and H.

\section{Structure of pre-ELM magnetic fluctuations}

Analyzing the toroidal structure of magnetic fluctuations can give further insight in the underlying instabilities causing them. Furthermore, comparing the frequencies of the detected $\partial B_{\mathrm{r}} / \partial t$ enables the determination of propagation velocities of the structures.

To measure the toroidal mode structure a toroidal array of magnetic pickup coils is installed in ASDEX Upgrade. Analyzing the relative phase shifts (and multiples of $2 \pi$ added/subtracted) in between the coils signals, one can determine the toroidal structure of the detected fluctuations, represented by the toroidal mode number $(n)$. It is important to consider that the pickup coils can have an intrinsic, frequency dependent phase response (due to inductances or shielding), especially at higher frequencies. For this reason, these intrinsic phases have to be taken into account for a reliable $n$ determination ${ }^{29}$. Recently, the method has been improved by statistically increasing the amount of data using ELM synchronization ${ }^{30}$, which enables the analysis of $n$ throughout the ELM cycle.

To compare the frequency resolved magnetic activity at the LFS midplane for the hydrogenic species, Figs. 5a and 5c present ELM synchronized frequency histograms for D and $\mathrm{H}$. At lower frequencies $(<40 \mathrm{kHz})$ the frequency histogram is dominated by a core mode in both presented cases. The post-ELM magnetic activity in the medium frequency range (40 to $200 \mathrm{kHz})$ during the $n_{\mathrm{e}}$ pedestal recovery $\left(\Delta t_{n_{\mathrm{e}}}\right.$, Fig. 4 ) is lower than in the other phases of the pedestal evolution. In $\mathrm{D}$ the fluctuations in this frequency range are more band-like than in $\mathrm{H}$, where they are rather broadband. After the recovery of the $T_{\mathrm{e}}$ pedestal $\left(\Delta t_{T_{\mathrm{e}}}\right)$ high frequency fluctuations between 300 and $400 \mathrm{kHz}$ set in. The preELM phases (between -2.1 and -0.1 ms relative to the ELM onset) are analyzed in detail by the ELM synchronized toroidal mode number histograms (Figs. $5 \mathrm{~b}$ and $5 \mathrm{~d}$ ). The sign of the mode numbers indicates their rotation direction. Positive $n$ represent a co-current (toroidal) or ion-diamagnetic (poloidal) propagation direction, whereas negative $n$ indicate a counter-current (toroidal) or electron-diamagnetic (poloidal) propagation direction. At lower frequencies the core mode has a clear $n=+1,+2$ structure for both plasmas. The 

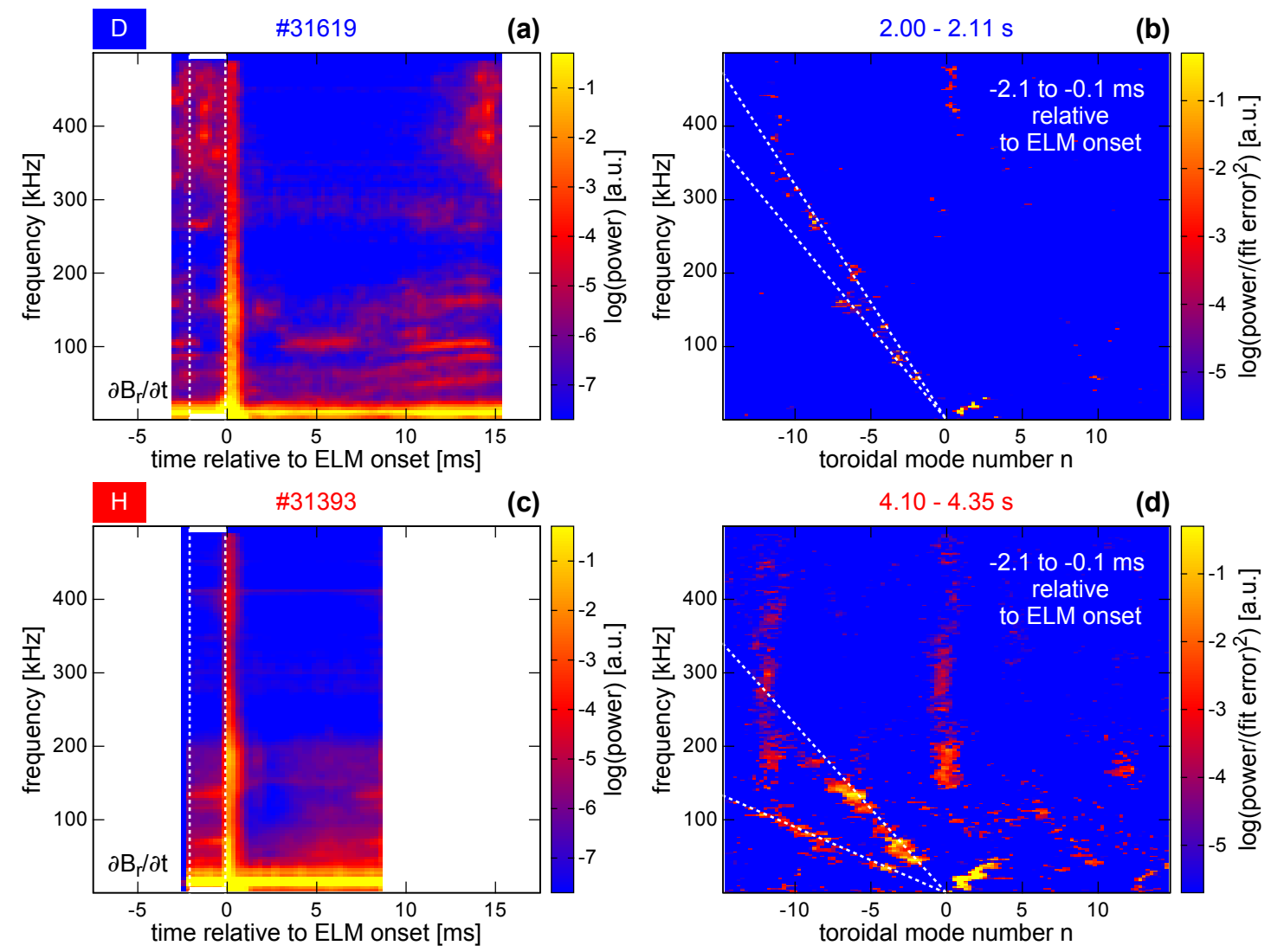

FIG. 5: Comparison of the magnetic fluctuations: ELM synchronized (a) f frequency and (b d toroidal mode number histograms (determined between -2.1 and $-0.1 \mathrm{~ms}$ relative to the ELM onset) for (a b $\mathrm{D}$ and (c) $\mathrm{d}$ ) $\mathrm{H}$. The white dashed lines in (a) indicate the time interval of the mode number determination and in (b.d) the two mode branches with different propagation velocity. Similar inter-ELM activity and corresponding toroidal structure can be seen in D and H. The different detected frequencies for similar $n$ correspond to different rotation velocities relative to the lab frame.

higher frequency range shows two mode branches with negative $n$ for $\mathrm{D}$ and $\mathrm{H}$ as it has been found previously in a D discharge $\mathrm{e}^{30}$. These are indicated by the white dashed lines, which inclination represent the rotation velocity relative to the lab frame. The different inclination means that the two branches rotate at different velocities. The shallower slopes of the branches in $\mathrm{H}$ in comparison to $\mathrm{D}$ indicate a lower rotation velocity. This can be explained by the different $n_{\mathrm{e}}$ profile in $\mathrm{H}$, since the $\mathrm{E} \times \mathrm{B}$ flow at the edge is proportional to $\nabla p_{\mathrm{i}} /\left(e \cdot n_{\mathrm{i}}\right)$. Assuming $\nabla n_{\mathrm{i}} / n_{\mathrm{i}} \approx \nabla n_{\mathrm{e}} / n_{\mathrm{e}}$, the shallower $\nabla n_{\mathrm{e}}$ in $\mathrm{H}$ leads to a lower background $\mathrm{E} \times \mathrm{B}$ velocity. 
Pedestal structure and inter-ELM evolution for different main ion species

It can be seen that the branches have similar structure in both main ion species. At medium frequencies $n$ is in the region of -3 to -8 . Consistent with the more broadband ELM synchronized frequency histograms in $\mathrm{H}$, the measured $n$ have less distinct peaks in the mode number range than in $\mathrm{D}$. The high frequency fluctuations are related to $n$ in the region of -11 in both main ion species.

The comparison of the pre-ELM structure of $\partial B_{\mathrm{r}} / \partial t$ reveals similar $n$ for both main ion species, which also supports that similar instabilities are present in these plasmas. The detected rotation velocities differ, since the $\mathrm{E} \times \mathrm{B}$ flow velocity is affected by the shallower density gradient in $\mathrm{H}$.

\section{HELIUM PLASMAS}

For further comparison to another main ion species, ${ }^{4}$ He plasmas were performed. Again a match of the pedestal $n_{\mathrm{e}}$ and $T_{\mathrm{e}}$ to the $\mathrm{D}$ and $\mathrm{H}$ references (see Section III A) was envisaged. However, due to the two electrons provided by a ${ }^{4} \mathrm{He}$ atom to the plasma, the main ion density $\left(n_{\mathrm{i}}\right)$ was lower in comparison to hydrogenic species at similar $n_{\mathrm{e}}$.

In ${ }^{4} \mathrm{He}$ several operational boundaries at ASDEX Upgrade have to be taken into account. For the density control only a small pumping rate by the turbomolecular pumps are available. This issue can be overcome by applying argon frosting 31 . It was decided to operate the NBI with $\mathrm{H}$ for these experiments, because ${ }^{4} \mathrm{He}$ NBI would suffer from a significant reduction of available $P_{\text {heat }}$ and no CXRS measurements for $T_{\mathrm{i}}$ would be possible. According to these operational limits, plasmas with a ${ }^{4} \mathrm{He}$ concentration in the region of $80 \%$ of the ion density were performed. Approximately $17 \% \mathrm{H}$ content is caused by the NBI and a $3 \%$ $\mathrm{D}$ concentration is due to residual gas released from the wall.

\section{A. Inter-ELM pedestal evolution}

In comparison to the $\mathrm{D}$ and $\mathrm{H}$ discharges higher $n_{\mathrm{e}}$ pedestals are present in this period (Fig. 6$)$ but $W_{\mathrm{MHD}}, f_{\mathrm{ELM}}$ and relative $\Delta W_{\mathrm{MHD}}$ are comparable to the $\mathrm{D}$ discharge. In Fig. 7 the evolution of $\nabla n_{\mathrm{e}}, \nabla T_{\mathrm{e}}$ and $\nabla T_{\mathrm{i}}$ at radial positions close to the separatrix $\left(\rho_{p o l} 0.98,0.99\right)$ are shown. Similar recovery phases as in the hydrogenic plasmas are identified. Again $n_{\mathrm{e}}$, respectively $\nabla n_{\mathrm{e}}$ recovers faster than $\nabla T_{\mathrm{e}}$. Remarkably, $\nabla T_{\mathrm{i}}$ does not follow the evolution 

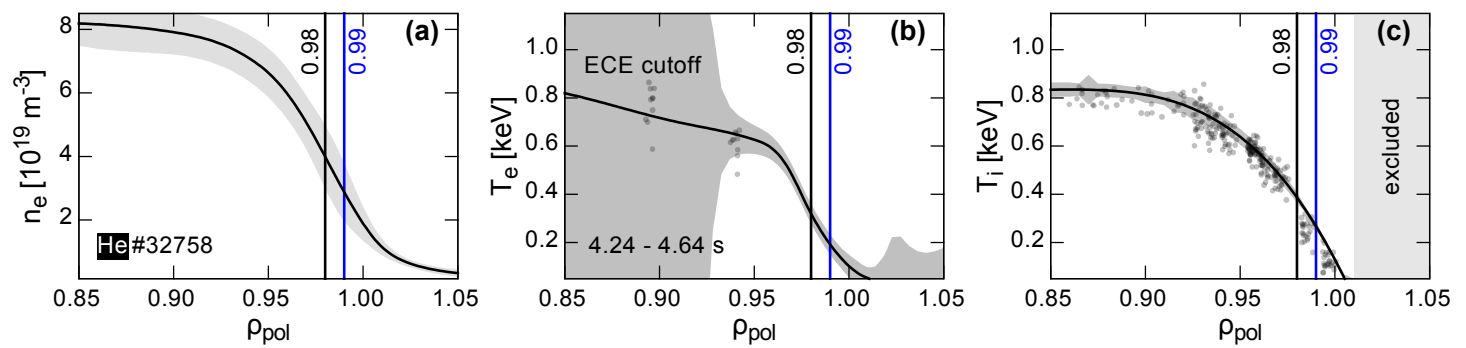

FIG. 6: Pedestal profiles in ${ }^{4} \mathrm{He}$ : (a) $n_{\mathrm{e}}$, (b) $T_{\mathrm{e}}$, (c) $T_{\mathrm{i}}$, averaged between -2 and $-1 \mathrm{~ms}$ relative to the ELM onset. The pedestal top $n_{\mathrm{e}}$ is roughly $50 \%$ higher than in the hydrogenic species (see Fig. 1), leading to a cutoff of the ECE inside $\rho_{\text {pol }}=0.95$.

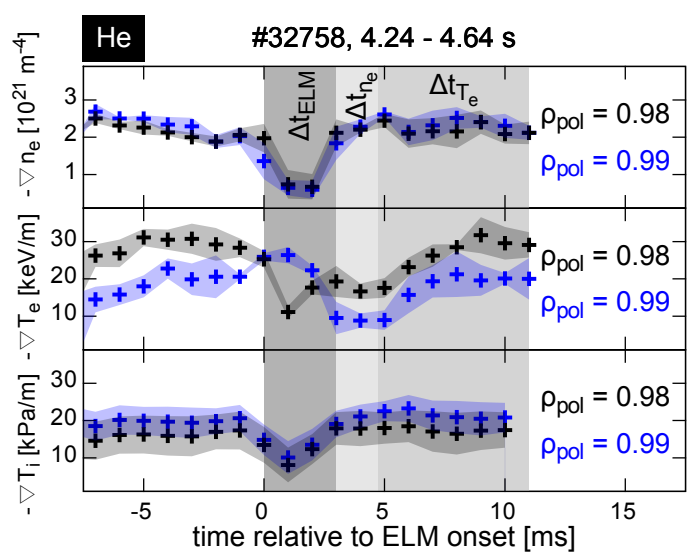

FIG. 7: Temporal evolution of the pedestal gradients in ${ }^{4} \mathrm{He}: \nabla n_{\mathrm{e}}, \nabla T_{\mathrm{e}}$ and $\nabla T_{\mathrm{i}}$ at two positions in the pedestal $\left(\rho_{p o l}=0.98,0.99\right)$. The recovery of $\nabla n_{\mathrm{e}}$ and $\nabla T_{\mathrm{i}}$ take place on similar timescales $\left(\Delta t_{n_{\mathrm{e}}}\right)$, whereas $\nabla T_{\mathrm{e}}$ is established later in the ELM cycle $\left(\Delta t_{T_{\mathrm{e}}}\right)$.

of $\nabla T_{\mathrm{e}}$ but rather is re-established after $\Delta t_{n_{\mathrm{e}}}$, which is the recovery timescale of $n_{\mathrm{e}}$. Such a behaviour was also suggested by the comparison of the neoclassically estimated current in the pedestal (using $T_{\mathrm{i}}$ equal to $T_{\mathrm{e}}$ ) and the current in the pedestal reconstructed from magnetic measurements $\sqrt{26}$, because the neoclassically estimated current evolves slower than the reconstructed one. More recently, the recovery behaviour of $T_{\mathrm{i}}$ has also been investigated in D plasmas, where impurities were puffed for fast $T_{\mathrm{i}}$ measurements ${ }^{32}$. It has also been found that the $T_{\mathrm{i}}$ pedestal recovers on similar time as the $n_{\mathrm{e}}$ pedestal. 

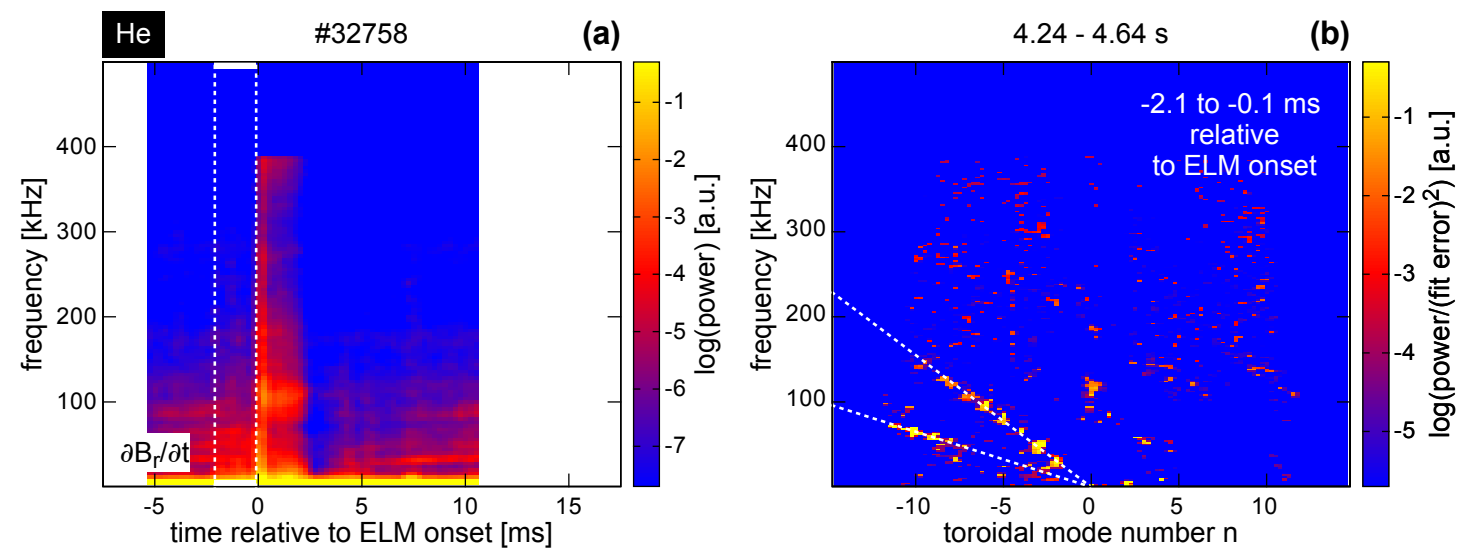

FIG. 8: Comparison of the magnetic fluctuations: ELM synchronized a frequency and (b) toroidal mode number histograms (determined between -2.1 and $-0.1 \mathrm{~ms}$ relative to the ELM onset) for ${ }^{4} \mathrm{He}$. The white dashed lines in (a) indicate the time interval of the mode number determination and in (b) the two mode branches with different propagation velocity. In comparison to $\mathrm{D}$ and $\mathrm{H}$ similar magnetic activity and $n$ are found at lower detected frequencies owing to the lower propagation velocity relative to the lab frame.

\section{B. Structure of pre-ELM magnetic fluctuations}

As it can be seen in the ELM synchronized frequency histograms there is much less medium and high frequency magnetic activity (100 to $400 \mathrm{kHz}$, Fig. 8 a) than in the investigated cases of D and $\mathrm{H}$. Also the frequency of an acting core mode is lower in ${ }^{4} \mathrm{He}$. Both are indications that the rotation velocities are lower in this case. The low core rotation can be explained by the lower NBI momentum input than e.g. in H (less NBI sources), whereas a lower poloidal edge rotation can be explained by a change in the $\nabla p_{\mathrm{i}} /\left(e \cdot n_{\mathrm{i}}\right)$ term.

During $\Delta t_{n_{\mathrm{e}}}$ a phase with very low magnetic activity is present, similar to the one observed in the hydrogenic plasmas. Later on in the ELM cycle well defined frequency bands develop, which last till the onset of the next ELM.

Analyzing the pre-ELM (determined between -2.1 and $-0.1 \mathrm{~ms}$ relative to the ELM onset) toroidal structure (Fig. 8 b two mode branches are found, similar to the one in D and $\mathrm{H}$. The contributing mode numbers are well defined and in the range of $n-2$ to -11 . As indicated by the white dashed lines the propagation velocities relative to the lab frame are lower than in the hydrogenic plasmas. This is in line with a lower rotation of the plasma owing to the previously discussed arguments. In summary, clear evidence is found that the 
Pedestal structure and inter-ELM evolution for different main ion species

same kind of instabilities as in hydrogenic plasmas are also present and dominant in the pedestal of ${ }^{4} \mathrm{He}$ plasmas with ELMs.

\section{SUMMARY AND DISCUSSION}

Pedestal matching experiments between D, H and ${ }^{4} \mathrm{He}$ plasmas were performed to compare the pedestal structure, stability and inter-ELM evolution. The overall behaviour of these discharges was different in the sense that different energy as well as particle confinement is observed. In $\mathrm{H}$ due to lower particle confinement a shallower $\nabla n_{\mathrm{e}}$ is found and a factor of approximately 2 higher heat flux through the pedestal is estimated in comparison to D. In ${ }^{4} \mathrm{He}$ due to the higher plasma dilution (lower $n_{\mathrm{i}}$ ) the $n_{\mathrm{e}}$ at the pedestal top is roughly $50 \%$ larger than for the hydrogenic references with similar $W_{\text {MHD }}$ and $T_{\text {e }}$ pedestal profiles.

The analyses of the inter-ELM $n_{\mathrm{e}}$ and $T_{\mathrm{e}}$ profile evolutions showed similar sequences in the pedestal recovery for D, $\mathrm{H}$ and ${ }^{4} \mathrm{He}$. After the ELM crash the $n_{\mathrm{e}}$ pedestal recovers first, then the $T_{\mathrm{e}}$ pedestal recovers. In ${ }^{4} \mathrm{He}$ detailed studies on the inter-ELM recovery of the $T_{\mathrm{i}}$ pedestal were made. These show that $T_{\mathrm{i}}$ recovers on the same timescale as $n_{\mathrm{e}}$, as suggested previously $^{26}$ and also observed in D plasmas with impurity seeding $\sqrt{32}$. The magnetic activity throughout the ELM cycle is comparable in all investigated main ions species. After the ELM, which causes large magnetic fluctuations a phase with very low activity is found, which duration corresponds to the recovery of the $n_{\mathrm{e}}$ pedestal. Later on the magnetic fluctuations with a sometimes well defined band structure set in, which continue till the next ELM crash. These have similar $n$ in the range of -3 to -8 in all presented cases. When the pedestals of $n_{\mathrm{e}}$ and $T_{\mathrm{e}}$ are recovered, fluctuations with $n$ in the region of $n-10$ to -11 set in. Furthermore, two mode branches with different propagation velocities relative to the lab frame can be identified. Comparing the different main ion species, changes in the edge profiles impact the $\mathrm{E} \times \mathrm{B}$ flow which can explain the different propagation velocity relative to the lab frame.

In conclusion, the experimental observations suggest that the pedestal stability can be sufficiently described by PB theory in the investigated hydrogenic species plasmas. The non-linear phase of the ELM crash could be affected since the ELM losses vary when the main isotope species is exchanged. The inter-ELM pedestal recovery behaviour as well as 
Pedestal structure and inter-ELM evolution for different main ion species

the identified magnetic signature and structure point into the direction that the sequence of dominant mechanisms in the pedestal evolution proceeds independently of the main ion species.

\section{ACKNOWLEDGEMENT}

F. M. Laggner is a fellow of the Friedrich Schiedel Foundation for Energy Technology. This work has been carried out within the framework of the EUROfusion Consortium and has received funding from the Euratom research and training programme 2014-2018 under grant agreement No 633053. The views and opinions expressed herein do not necessarily reflect those of the European Commission.

\section{REFERENCES}

${ }^{1}$ R. Aymar, P. Barabaschi, Y. Shimomura, and the ITER Team, Plasma Physics and Controlled Fusion 44, 519 (2002).

${ }^{2}$ H. Biglari, P. H. Diamond, and P. W. Terry, Physics of Fluids B-Plasma Physics 2, 1 $(1990)$.

${ }^{3}$ J. W. Connor and H. R. Wilson, Plasma Physics and Controlled Fusion 42, R1 (2000).

${ }^{4}$ D. Boucher and V. Mukhovatov, Plasma Physics and Controlled Fusion 38, 1225 (1996).

${ }^{5}$ Y. R. Martin, T. Takizuka, and ITPA CDBM H-mode Threshold Database Working Team, 11th Iaea Technical Meeting on H-Mode Physics and Transport Barriers 123, 012033 (2008).

${ }^{6}$ F. Ryter, M. Cavedon, T. Happel, R. M. McDermott, E. Viezzer, G. D. Conway, R. Fischer,

B. Kurzan, T. Putterich, G. Tardini, M. Willensdorfer, and A. U. Team, Plasma Physics and Controlled Fusion 58, 014007 (2016).

${ }^{7}$ M. Bessenrodt-Weberpals, F. Wagner, O. Gehre, L. Giannone, J. V. Hofmann, A. Kallenbach, K. McCormick, V. Mertens, H. D. Murmann, F. Ryter, B. D. Scott, G. Siller, F. X. Soldner, A. Stabler, K. H. Steuer, U. Stroth, N. Tsois, H. Verbeek, and H. Zoohm, Nuclear Fusion 33, 1205 (1993).

${ }^{8}$ F. Wagner and U. Stroth, Plasma Physics and Controlled Fusion 35, 1321 (1993). 
Pedestal structure and inter-ELM evolution for different main ion species

${ }^{9}$ H. Urano, T. Takizuka, M. Kikuchi, T. Nakano, N. Hayashi, N. Oyama, and Y. Kamada, Physical Review Letters 109 (2012), 10.1103/PhysRevLett.109.125001.

${ }^{10}$ P. A. Schneider, L. B. Orte, A. Burckhart, M. G. Dunne, C. Fuchs, A. Gude, B. Kurzan, W. Suttrop, E. Wolfrum, and the ASDEX Upgrade Team, Plasma Physics and Controlled Fusion 57, 014029 (2015).

${ }^{11}$ V. P. Bhatnagar, J. Lingertat, R. Barnsley, P. Breger, J. P. Christiansen, S. Clement, J. G. Cordey, S. J. Davies, J. K. Ehrenberg, L. G. Eriksson, G. M. Fishpool, P. J. Harbour, L. D. Horton, J. Jacquinot, H. J. Jackel, K. Lawson, C. G. Lowry, C. F. Maggi, G. F. Matthews, R. D. Monk, D. P. O’Brien, V. V. Parail, E. Righi, G. Saibene, R. Sartori, B. Schunke, A. C. C. Sips, M. F. Stamp, D. F. H. Start, and K. Thomsen, Nuclear Fusion 39, 353 (1999).

${ }^{12}$ A. Scarabosio, C. Fuchs, A. Herrmann, E. Wolfrum, and A. U. Team, Journal of Nuclear Materials 415, S877 (2011).

${ }^{13}$ C. McDonald, J. G. Cordey, E. Righi, F. Ryter, G. Saibene, R. Sartori, B. Alper, M. Becoulet, J. Brzozowski, I. Coffey, M. de Baar, P. de Vries, K. Erents, W. Fundamenski, C. Giroud, I. Jenkins, A. Loarte, P. J. Lomas, G. P. Maddison, J. Mailloux, A. Murari, J. Ongena, J. Rapp, R. A. Pitts, M. Stamp, J. Strachan, W. Suttrop, and JET EFDA Contributors, Plasma Physics and Controlled Fusion 46, 519 (2004).

${ }^{14}$ H. Urano, T. Takizuka, N. Aiba, M. Kikuchi, T. Nakano, T. Fujita, N. Oyama, Y. Kamada, N. Hayashi, and the JT-60 Team, Nuclear Fusion 53, 083003 (2013).

${ }^{15}$ H. Urano, Nuclear Fusion 54, 116001 (2014).

${ }^{16}$ E. Wolfrum, E. Viezzer, A. Burckhart, M. G. Dunne, P. A. Schneider, M. Willensdorfer, E. Fable, R. Fischer, D. Hatch, F. Jenko, B. Kurzan, P. Manz, S. K. Rathgeber, and the ASDEX Upgrade Team, Nuclear Fusion 55, 053017 (2015).

${ }^{17}$ M. Willensdorfer, G. Birkenmeier, R. Fischer, F. M. Laggner, E. Wolfrum, G. Veres, F. Aumayr, D. Carralero, L. Guimarais, B. Kurzan, and the ASDEX Upgrade Team, Plasma Physics and Controlled Fusion 56, 025008 (2014).

${ }^{18}$ A. Mlynek, M. Reich, L. Giannone, W. Treutterer, K. Behler, H. Blank, A. Buhler, R. Cole, H. Eixenberger, R. Fischer, A. Lohs, K. Luddecke, R. Merkel, G. Neu, F. Ryter, D. Zasche, and the ASDEX Upgrade Team, Nuclear Fusion 51, 043002 (2011).

${ }^{19}$ W. Suttrop, A. G. Peeters, the ASDEX Upgrade Team, and the NBI group, "Practical limitations to plasma edge electron temperature measurements by radiometry of electron 
Pedestal structure and inter-ELM evolution for different main ion species

cyclotron emission," Tech. Rep. (1996).

${ }^{20}$ R. Fischer, C. J. Fuchs, B. Kurzan, W. Suttrop, E. Wolfrum, and the ASDEX Upgrade Team, Fusion Science and Technology 58, 675 (2010).

${ }^{21}$ S. K. Rathgeber, L. Barrera, T. Eich, R. Fischer, B. Nold, W. Suttrop, M. Willensdorfer, E. Wolfrum, and A. U. Team, Plasma Physics and Controlled Fusion 55, 025004 (2013).

${ }^{22}$ B. Kurzan, H. Murmann, H. Salzmann, and the ASDEX Upgrade Team, Review of Scientific Instruments 72, 1111 (2001)

${ }^{23}$ E. Viezzer, T. Putterich, R. Dux, R. M. McDermott, and the ASDEX Upgrade Team, Rev Sci Instrum 83, 103501 (2012)

${ }^{24}$ M. Cavedon, T. Putterich, E. Viezzer, R. M. Dux, B. Geiger, R. McDermott, H. Meyer, U. Stroth, and the ASDEX Upgrade Team, In preparation for Review of Scientific Instruments (2016).

${ }^{25}$ M. Dunne, L. Frassinetti, M. N. A. Beurskens, M. Cavedon, S. Fietz, R. Fischer, L. Giannone, G. T. A. Huijsmans, B. Kurzan, F. M. Laggner, P. J. McCarthy, R. M. McDermott, G. Tardini, E. Viezzer, M. Willensdorfer, E. Wolfrum, E. M. Team, and the ASDEX Upgrade Team, Accepted in Plasma Physics and Controlled Fusion (2016).

${ }^{26}$ M. G. Dunne, P. J. McCarthy, E. Wolfrum, R. Fischer, L. Giannone, A. Burckhart, and the ASDEX Upgrade Team, Nuclear Fusion 52, 123014 (2012).

${ }^{27}$ F. M. Laggner, E. Wolfrum, M. Cavedon, F. Mink, E. Viezzer, M. G. Dunne, P. Manz, H. Doerk, G. Birkenmeier, R. Fischer, S. Fietz, M. Maraschek, M. Willensdorfer, F. Aumayr, and the ASDEX Upgrade Team, Plasma Physics and Controlled Fusion 58, 065005 (2016).

${ }^{28}$ A. Burckhart, E. Wolfrum, R. Fischer, K. Lackner, H. Zohm, and the ASDEX Upgrade Team, Plasma Physics and Controlled Fusion 52, 105010 (2010).

${ }^{29}$ L. Horvth, P. Z. Poloskei, G. Papp, M. Maraschek, K. H. Schuhbeck, G. I. Pokol, the EUROfusion MST1 Team, and the ASDEX Upgrade Team, Plasma Physics and Controlled Fusion 57, 125005 (2015)

${ }^{30}$ F. Mink, E. Wolfrum, M. Maraschek, H. Zohm, L. Horvth, F. M. Laggner, P. Manz, E. Viezzer, U. Stroth, and the ASDEX Upgrade Team, Plasma Physics and Controlled Fusion 58, 125013 (2016).

${ }^{31}$ M. R. Wade, D. L. Hillis, J. T. Hogan, M. A. Mahdavi, R. Maingi, W. P. West, N. H. Brooks, K. H. Burrell, R. J. Groebner, G. L. Jackson, C. C. Klepper, G. Laughon, M. M. 
Pedestal structure and inter-ELM evolution for different main ion species

Menon, and P. K. Mioduszewski, Physical Review Letters 74, 2702 (1995).

${ }^{32} \mathrm{M}$. Cavedon and the ASDEX Upgrade Team, In preparation for Plasma Physics and Controlled Fusion (2016). 